\title{
3. The effects of alternating electric fields in glioblastoma: current evidence on therapeutic mechanisms and clinical outcomes
}

\begin{abstract}
Azeem A. Rehman, BS, ${ }^{1}$ Kevin B. Elmore, BS, ${ }^{1}$ and Tobias A. Mattei, MD ${ }^{2}$
1 University of Illinois College of Medicine at Peoria, Illinois; and 2Department of Neurosurgery, Brain \& Spine Center, Invision Health, Buffalo, New York

Glioblastoma is both the most common and most lethal primary CNS malignancy in adults, accounting for $45.6 \%$ of all malignant CNS tumors, with a 5-year survival rate of only 5.0\%, despite the utilization of multimodal therapy including resection, chemotherapy, and radiation. Currently available treatment options for glioblastoma often remain limited, offering brief periods of improved survival, but with substantial side effects. As such, improvements in current treatment strategies or, more likely, the implementation of novel strategies altogether are warranted. In this topic review, the authors provide a comprehensive review on the potential of alternating electric fields (AEFs) in the treatment of glioblastoma. Alternating electric fields_-also known as tumor-treating fields (TTFs) -represent an entirely original therapeutic modality with preliminary studies suggesting comparable, and at times improved, efficacy to standard chemotherapeutic agents in the treatment of recurrent glioblastoma. A recent multicenter, Phase III, randomized clinical trial comparing NovoTTF-100A monotherapy to physician's best choice chemotherapy in patients with recurrent glioblastoma revealed that AEFs have similar efficacy to standard chemotherapeutic agents with a more favorable side-effects profile and improved quality of life. In particular, AEFs were shown to have limited systemic adverse effects, with the most common side effect being contact dermatitis on the scalp at the sites of transducer placement. This study prompted FDA approval of the NovoTTF-100A system in April 2011 as a standalone therapy for treatment of recurrent glioblastoma refractory to surgical and radiation treatment. In addition to discussing the available clinical evidence regarding the utilization of AEFs in glioblastoma, this article provides essential information regarding the supposed therapeutic mechanism as well as modes of potential tumor resistance to such novel therapy, delineating future perspectives regarding basic science research on the issue.
\end{abstract}

http://thejns.org/doi/abs/10.3171/2015.1.FOCUS14742

KEY WORDS tumor-treating fields; alternating electric fields; glioblastoma; neurooncology; progression-free survival; overall survival

$\mathrm{P}$ RIMARY and metastatic brain tumors comprise a heterogeneous group of CNS malignancies, varying in histological features, ${ }^{7}$ phenotypic presentation, cell origin, and tumor grade as designated by the WHO. ${ }^{38}$ The incidence of primary brain malignancies has been increasing, with an estimated 23,380 diagnoses and 14,320 deaths in 2014 attributed to CNS malignancies. ${ }^{61}$ Despite technological advances in neuroimaging, which have enabled earlier detection and closer monitoring of treatment effects, these tumors continue to be associated with significant mortality and morbidity. Glioblastoma is both the most common and most lethal primary CNS malignancy in adults, accounting for $45.6 \%$ of all malignant CNS tumors ${ }^{49}$ presenting a dismal 5-year survival rate of only $5.0 \%$ despite the utilization of multimodal therapy. ${ }^{63}$

Management of CNS tumors involves a complex multidisciplinary team of neurosurgeons, neurooncologists, and radiation oncologists, using standard strategies consisting of resection, chemotherapy, and radiation. Specific treatment regimens are usually tailored to patients depending on age, prognostic status, tumor location, tumor type, tumor grade, and staging. Aggressive surgery with complete

ABBREVIATIONS $\mathrm{AEF}=$ alternating electric field; OS $=$ overall survival; $\mathrm{PFS}=$ progression-free survival; PFS6 $=$ PFS at 6 months; TTF $=$ tumor-treating field . SUBMITTED October 31, 2014. ACCEPTED January 9, 2015.

INCLUDE WHEN CITING DOI: 10.3171/2015.1.FOCUS14742.

DISCLOSURE The authors report no conflict of interest concerning the materials or methods used in this study or the findings specified in this paper. 
macroscopic resection for glioblastoma has been shown to improve overall patient survival $;{ }^{36}$ however, complete microscopic removal of the tumor is challenging given its diffuse and infiltrative nature, with nearly all glioblastomas eventually recurring. Thus, adjuvant therapy is routinely used to improve both the progression-free survival (PFS) and overall survival (OS). Traditional adjuvant therapy following glioblastoma resection involves fractionated radiation therapy, which has been shown to improve overall survival by $3-6$ months as compared with supportive care $^{34,76}$ For younger patients with good postoperative performance, temozolomide is often used in combination with radiotherapy and has been shown to improve survival by $2-5$ months in clinical trials. ${ }^{9,25,64}$ Although some studies have suggested that carmustine wafers (Gliadel, Eisai) placed at the time of surgery may also improve survival in patients with recurrent high-grade gliomas, such a therapeutic modality involves a significant additional cost and its use is not widespread either in the US or Europe. ${ }^{6}$ Many drugs are available as alternative and salvage chemotherapy, most notably bevacizumab (Avastin, Genetech), which has been shown to slow radiological progression and improve survival in recurrent glioblastoma. . $^{15,33,74}$

Despite their efficacy, chemotherapeutic agents are often associated with noteworthy side effects. For instance, patients receiving temozolomide have been shown to often experience hair loss, nausea, vomiting, headaches, fatigue, and anorexia., ${ }^{2,83}$ Additionally, temozolomide may cause lymphocytopenia leading to opportunistic infection, ${ }^{32}$ with some patients requiring prophylaxis against pneumonia by Pneumocystis carinii when chemotherapy is given in combination with radiation therapy. Additionally, toxicity has been reported when carmustine wafers are supplemented with other chemotherapeutic agents, but this finding has not been consistent across different studies. ${ }^{10,40,57}$ Finally, bevacizumab has been associated with serious hemorrhagic events, ${ }^{23}$ hypertension, ${ }^{66}$ impaired wound healing, colonic perforation, thromboembolism, and renal failure. . $^{47,81}$

At present, treatment options for glioblastoma remain limited, offering only brief periods of improved survival. In a landmark clinical trial for glioblastoma treatment, Stupp et al. in 2005 noted mean PFS to be just 6.9 months, despite the combination of radiotherapy with adjuvant temozolomide following maximal surgical debulking. ${ }^{64}$ Given the substantial side-effect profiles of chemotherapy, improvements in current available treatment strategies or, more likely, the implementation of novel strategies altogether are warranted. The field of bioelectromagnetics offers a potential avenue of innovative approaches by exploring the effects of electromagnetic fields on biological systems, including cells and tissues. The potential of electric fields in particular has already been adapted for various applications, including osteogenesis stimulation for nonhealing fractures, ${ }^{3}$ wound healing ${ }^{84}$ and neural cell regeneration, ${ }^{62}$ as well as symptomatic relief in osteoarthritis ${ }^{69}$ Most recently, the technology has been employed as an anti-cancer strategy.

In April of 2011, the FDA approved the NovoTTF-100A system (Novocure, Inc.) as a standalone therapy for treating recurrent glioblastoma refractory to surgical and ra- diation treatment. ${ }^{72}$ Utilizing a portable, battery-operated device, alternating electric fields (AEFs) are transmitted to the tumor via disposable surface electrodes engaged indirectly on the skin using a conductive hydrogel (Fig. 1). These electrodes (Fig. 2) are placed after head mapping with a series of MR images, and the AEFs are continuously applied over an extended period, at least 4 weeks. These AEFs-also known as tumor-treating fields (TTFs) - represent a novel treatment option for high-grade gliomas that categorically differs from the traditional surgical, chemotherapeutic, and radiation approaches.

\section{Mechanism of Action}

Electric fields are forces capable of acting on charged particles, creating characteristic movements dependent on the strength of the applied field and the magnitude of the charged particle upon which they act. Cells inherently contain electrically charged molecules, including DNA ${ }^{16}$ and proteins ${ }^{21}$ which are subject to forces exerted by electric fields. Thus, in the setting of AEFs, these charged molecules oscillate at a rate dependent on the frequency, intensity, and vector of the applied electric field. ${ }^{4}$ If this field is nonuniform, dielectric molecules can become suspended along the electric fields and separate from each other, a phenomenon known as dielectrophoresis. ${ }^{17}$

Although the precise molecular effects of AEFs still remain under investigation, the most accepted pathophysiological explanation regarding their therapeutic effects on cancer relies on the effects of the AEFs upon the molecular machinery responsible for mitosis, more specifically, the microtubules. These components of the cytoskeleton are composed of tubulin subunits, consisting of globular proteins with substantial polarity that guide chromosomal

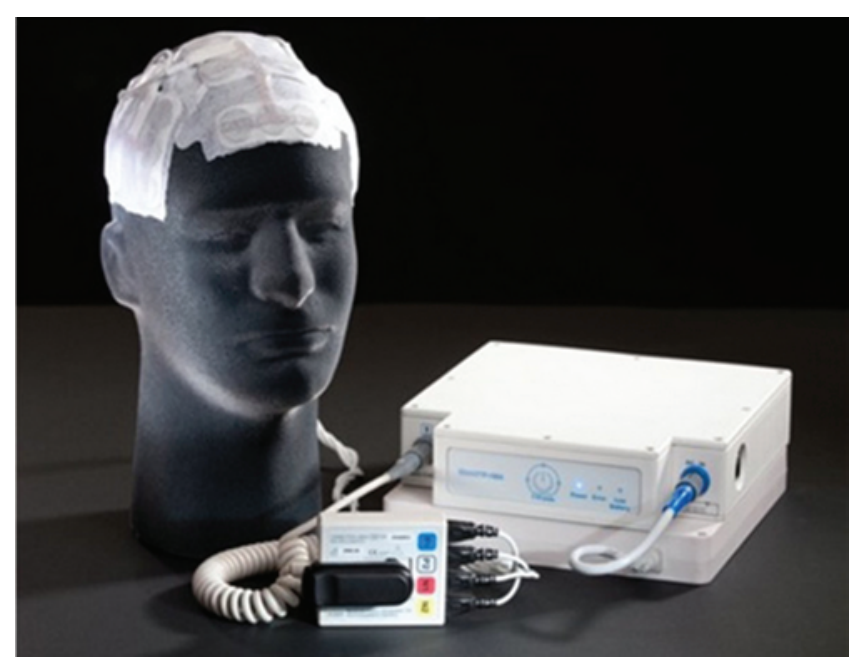

FIG. 1. The NovoTTF-100A system, consisting of noninvasive transducers that are attached to the scalp through a hydrogel interface. These transducers are connected to a portable battery and electric field generator. Reprinted with permission from Turner SG, Gergel T, Wu H, Lacroix M, Toms SA: The effect of field strength on glioblastoma multiforme response in patients treated with the NovoTTF-100A system. World J Surg Oncol 12:162, 2014. DOI:10.1186/1477-7819-12-162, http://www. wjso.com/content/12/1/162. No modification performed in original figure. Material available under Public License: Creative Commons license 4.0 (https://creativecommons.org/licenses/by/4.0/legalcode). 


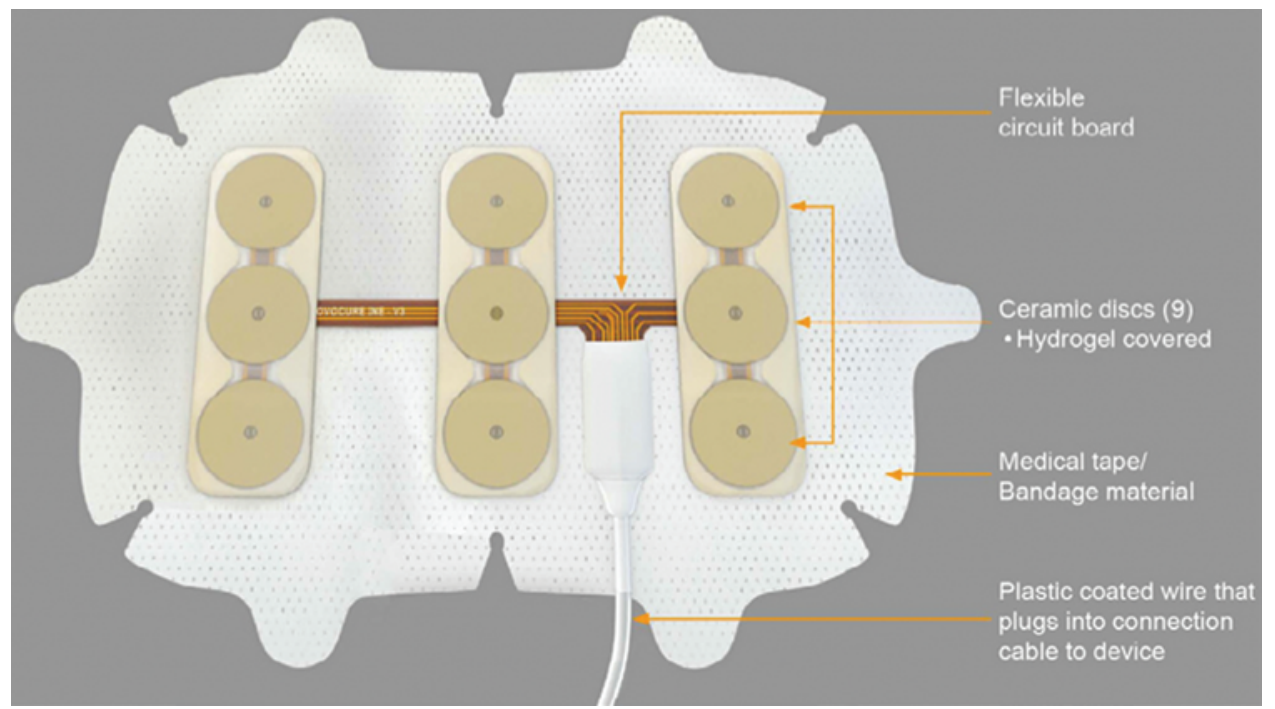

FIG. 2. The NovoTTF-100A system transducer array apparatus, which is placed directly on the scalp. Republished with permission of W.B./Saunders Co. from "Characterization and management of dermatologic adverse events with the NovoTTF-100A System, a novel anti-mitotic electric field device for the treatment of recurrent glioblastoma," Lacouture et al, Semin Oncol 41 Suppl 4:S1S14, 2014. Permission conveyed through Copyright Clearance Center, Inc.

aggregation and segregation. ${ }^{41}$ The inherent polarity of the tubulin subunits makes them sensitive to AEFs, which, by affecting their polymerization, ultimately disrupts the formation of the mitotic spindle apparatus, prolonging the mitotic process. Such effects have already been elegantly demonstrated using microphotography. ${ }^{29,37}$

In addition, following mitosis the process of cytokinesis involves a cleavage furrow undergoing ingression secondary to the action of a contractile ring, ultimately resulting in the formation of two separate cells. Interestingly, this cleavage furrow is composed of a high-density electric field region, capable of exerting significant force..$^{29,85}$ Upon exposure to AEFs, polar molecules and organelles preferentially migrate to higher field intensities. This phenomenon ultimately results in the movement of charged molecules toward the cleavage furrow, disrupting the intracellular apparatus and ultimately leading to plasma membrane blebbing, a common phenomenon observed in physiological apoptosis. ${ }^{29}$ As a result of such cellular effects, AEFs have been shown to selectively target tumor cells undergoing active mitosis and cytokinesis, while sparing nondividing cells, particularly surrounding normal neuronal and glial cells.

In addition to the above-described effects of AEFs on mitosis and cytokinesis, alternative modes of action have also been documented. AEFs have been shown to alter the alignment of negatively charged DNA molecules, thus limiting the DNA repair response in gliomas and breast cancer cell lines. ${ }^{19}$ This finding is consistent with findings from a meta-analysis assessing the effects of AEFs on a variety of cancer cell lines, which revealed alterations in genetic processes that regulate DNA replication and repair. ${ }^{20}$ This particular mechanism is especially relevant when AEFs are used alongside radiation therapy, which has been shown to induce DNA damage in cancer cells by the use of focused ionizing radiation. Furthermore, it has also been demonstrated that upon exposure to AEFs, malignant melanoma cell lines exhibit downregulation of CD34, an essential marker of increased tumor vessel density. ${ }^{8}$ Conversely, a prior study had reported that electric fields, in fact, promote angiogenesis. ${ }^{58}$ Ultimately, electric fields have also been shown to alter blood circulation in vessels of variable sizes, ${ }^{68}$ suggesting potential applications in highly vascularized solid tumors. Nuccitelli et al. reported that, in addition to decreasing tumor blood flow, application of AEFs is able to initiate pyknosis in melanoma cells, resulting in an overall $90 \%$ reduction in tumor size. ${ }^{48}$

Lastly, AEFs have also been shown to alter membrane permeation, leading to eventual necrosis and apoptosis of cancer cells. ${ }^{67}$ This particular finding provides a possible therapeutic mechanism that explains the observed synergistic effects of AEFs and chemotherapy observed in some patients. Ultimately, future research using novel forms of biosensors will likely provide a better understanding of how electric fields of varying intensities and frequencies may affect cancerous and noncancerous cells. For example, researchers have already been able to shed light on morphological and cell viability changes in ovarian cancer cells upon exposure to AEFs by using an impedance-based biosensor complemented with cell labeling and fluorescence microscopy. ${ }^{24}$

\section{Preclinical and Pilot Glioblastoma Studies}

Beginning in 2004, a growing number of studies reporting the result of in vitro, in vivo, and pilot clinical studies have shed light on the therapeutic potential of AEFs in the treatment of a variety of cancer types (Table 1). In the first study of its type, Kirson et al. (2004) reported that AEFs were not only able to inhibit cell proliferation in a variety of cancer cell lines, but also reduce growth and initiate destruction of malignant melanoma and adenocarcinoma in murine models. ${ }^{29}$ These initial in vivo experiments suggested the possibility of delivering AEFs to the 
TABLE 1. Overview of clinical studies involving TTF and patients with glioblastoma

\begin{tabular}{|c|c|c|c|c|c|c|c|c|c|c|c|}
\hline \multirow[b]{2}{*}{$\begin{array}{l}\text { Authors } \\
\text { \& Year }\end{array}$} & \multirow[b]{2}{*}{ Study Description } & \multirow[b]{2}{*}{$\begin{array}{l}\text { No. of } \\
\text { Pts }\end{array}$} & \multicolumn{3}{|c|}{ Median OS (mos) } & \multicolumn{3}{|c|}{ Median PFS6 (\%) } & \multicolumn{3}{|c|}{$\begin{array}{c}\text { Median Time to Disease } \\
\text { Progression (wks) }\end{array}$} \\
\hline & & & TTF & $\begin{array}{l}\text { Chemo } \\
\text { Agent }\end{array}$ & $\begin{array}{c}p \\
\text { Value }\end{array}$ & TTF & Chemo & $\begin{array}{c}\mathrm{p} \\
\text { Value }\end{array}$ & TTF & $\begin{array}{l}\text { Chemo } \\
\text { Agent }\end{array}$ & $\begin{array}{c}p \\
\text { Value }\end{array}$ \\
\hline $\begin{array}{l}\text { Kirson et al., } \\
2007\end{array}$ & $\begin{array}{l}\text { Pilot study of TTF monotherapy for temoz- } \\
\text { olomide-refractory glioblastoma }\end{array}$ & 10 & 14.5 & $6.8^{*}$ & NR & $50 \%$ & $15.3 \% *$ & NR & 26.1 & $9.5^{*}$ & NR \\
\hline $\begin{array}{l}\text { Kirson et al., } \\
2009\end{array}$ & $\begin{array}{l}\text { Pilot study of TTF w/ temozolomide vs } \\
\text { temozolomide monotherapy for newly } \\
\text { diagnosed glioblastoma }\end{array}$ & 10 & $>39 \dagger$ & $14.7^{*}$ & 0.00018 & $100 \% \dagger$ & $60.0 \% *$ & NR & $155 \dagger$ & $31^{*}$ & 0.0002 \\
\hline $\begin{array}{l}\text { Stupp et al., } \\
2012\end{array}$ & $\begin{array}{l}\text { Phase III clinical trial of TTF vs physician's } \\
\text { best choice chemo }\end{array}$ & 237 & 6.6 & 6.0 & 0.27 & $21.4 \%$ & $15.1 \%$ & 0.13 & 9.4 & 9.0 & 0.16 \\
\hline
\end{tabular}

Chemo = chemotherapy; NR = not reported by investigators; $p$ ts = patients.

* Reported historical data for comparison purposes.

$\dagger$ Includes adjuvant temozolomide therapy.

tumor through insulated, noninvasive electrodes. Notably, the optimal AEF frequency varied across cancer cell lines, with $200 \mathrm{kHz}$ being the optimal frequency for the treatment of gliomas while frequencies around $100 \mathrm{kHz}$ were the most effective against melanoma. These findings suggested the necessity of "fine-tuning" the application of AEFs to each specific tumor line. A supplementary investigation by the same authors replicated these significant findings while incorporating additional cancer cell lines and animal models, including intracranial gliomas. ${ }^{28}$

These initial results prompted a single-arm pilot study that involved the application of AEFs in monotherapy in 10 patients diagnosed with recurring glioblastoma. In addition to improving the time to disease progression (median 26.1 vs 9.5 weeks, respectively) and PFS at 6 months (PFS6; 50\% vs $15.3 \%$, respectively), this study ${ }^{28}$ also demonstrated a significant increase in OS when compared with historical controls (median 62.2 vs 29.3 weeks, respectively). The device was well tolerated, with the most frequent adverse effect being a moderate contact dermatitis in 9 of the 10 patients on the scalp at the site of electrode placement, which had significant relief following topical steroid administration. No hematological, gastrointestinal, neurological, or cardiac side effects were documented.

Following this study, in 2009 the same group investigated the use of adjuvant AEF application in the context of chemotherapy, exploring possible synergistic efficacy and side-effect profiles. ${ }^{30}$ When combined with paclitaxel, doxorubicin, cyclophosphamide, and dacarbazine, AEFs exhibited synergistic effects on limiting the growth of human glioma and breast carcinoma cell lines in vitro. A notable dose reduction (1-3 orders of magnitude) of all types of chemotherapeutic agents was possible while maintaining similar efficacy. Comparable results were observed in rabbits implanted with tumor tissue and treated with a combination of paclitaxel and AEFs. An additional pilot clinical trial was also initiated, involving a separate cohort composed of 10 patients. This second cohort involved patients recently diagnosed with glioblastoma who were treated with a combination of AEFs and temozolomide. In comparison with matched historical controls treated solely with temozolomide, the combination therapy (AEFs and temozolomide) not only increased median PFS (155 vs 31 weeks, respectively) but also improved median OS (> 39 vs 14.7 months, respectively). Again, the only side effect associated with AEFs was contact dermatitis noted in 18 of the 20 patients, which could be alleviated with topical corticosteroids and rotation of the surface electrodes. This particular study was the first clinical study to suggest that a combination therapy of AEFs and chemotherapy may potentially improve the outcomes of patients with glioblastoma. Moreover, according to previous studies, the incorporation of AEFs might enable a dose reduction of chemotherapeutic agents while maintaining treatment efficacy. Because very little systemic toxicity has been associated with AEFs, this multimodal treatment would likely lead to improved quality of life in such a patient population.

\section{Phase III Randomized Trial}

The encouraging results from the aforementioned studies prompted a multicenter, Phase III, prospective randomized clinical trial, comparing the NovoTTF-100A monotherapy to physician's best choice chemotherapy in patients with recurrent glioblastoma. ${ }^{65}$ The median age of the 237 selected patients was 54 years, with 120 cases assigned to AEF monotherapy and 117 assigned to chemotherapy, either as a single agent or a combination of agents. The study found that there was similar efficacy between treatment with AEFs and chemotherapy. No statistically significant difference in median OS (6.6 vs 6.0 months, $p$ $=0.27)$, PFS6 (21.4\% vs $15.1 \%, p=0.13)$, radiological response rate $(14 \%$ vs $9.6 \%, \mathrm{p}=0.19)$ or 1 -year survival rate ( $20 \%$ vs $20 \%, p=0.13$ ) was noted between the AEF and the chemotherapy cohorts.

Although this study failed to demonstrate an improvement in survival by AEFs, adverse effects were significantly lower in the AEF group. Within the AEF cohort, contact dermatitis occurred in $16 \%$ of the patients and responded well to topical steroids. Additionally, severe adverse events occurred less frequently in the AEF-treated compared with the chemotherapy-treated patients (6\% vs $16 \%, \mathrm{p}=0.022)$. Moreover, longitudinal quality of life questionnaires predominantly favored AEF therapy, including better cognitive and emotional function, less pain, and reduced fatigue. 
To summarize, this landmark clinical trial revealed that AEFs have similar efficacy to standard chemotherapeutic agents in patients with recurring glioblastoma, with a more favorable side-effect profile and quality of life. As a result, AEFs received FDA approval for the monotherapy treatment of adult patients with recurrent glioblastoma.

\section{Post-Hoc Subgroup Analyses}

Following this clinical trial, numerous post-hoc studies were conducted, ${ }^{65}$ as summarized in Table 2 . These subgroup analyses suggest that in certain populations, AEF therapy may, in fact, be more efficacious when compared with conventional chemotherapy regimens. For example, it has been shown that patients with favorable prognostic characteristics (such as age less than 60 years or Karnofsky Performance Scale score greater than $80 \%$ ), as well as patients with tumors refractory to bevacizumab treatment, had significantly greater OS with AEF therapy in comparison with standard chemotherapy. ${ }^{26,27,53}$ Moreover, when excluding patients who did not receive a complete cycle of treatment with AEFs, patients in the AEF cohort showed improved survival in comparison with physician's best choice chemotherapy. ${ }^{80} \mathrm{~A}$ survival benefit was also noted when comparing AEF monotherapy to those patients receiving bevacizumab. ${ }^{54}$ Interestingly, certain tumor characteristics were also associated with improved OS with AEFs, including prior low-grade glioma, refractory response to bevacizumab, and tumor size greater than $18 \mathrm{~cm}^{2} .26,27$

\section{Case Reports}

Several case reports have also been published, demonstrating variable individual responses to AEF therapy.
For example, continued enhancement of cystic lesions was initially observed despite AEF administration; however, a delayed inhibitory response was noted, suggesting that continued treatment with AEFs may still be reasonable despite preliminary tumor growth. ${ }^{56,73,75}$ AEF therapy in these cases resulted in long-term survival benefits, including anecdotal reports of patients with glioblastoma or recurrent glioblastoma treated with AEF monotherapy who did not require any further follow-up therapy for approximately 6-7 years. Other case reports have described radiological benefits for AEF therapy in combination therapy with bevacizumab despite prior bevacizumab resistance ${ }^{11}$ or bevacizumab plus temozolomide resistance. ${ }^{79}$

\section{Compliance With AEF Therapy}

Perhaps the greatest limitation to the efficacy of AEF therapy is the challenge associated with patient compliance. Unlike chemotherapy, AEF therapy involves a physical, nonchemical modality, with no associated halflife. Hence, the antitumor effects are immediately halted once the AEF therapy is stopped, explaining the need for continuous application throughout the day over extended periods. A kinetic modeling study proposed that at least 4 weeks of continuous AEF therapy is needed to achieve a substantial reversal of tumor growth. ${ }^{31}$ As such, the treatment with AEFs requires a substantial commitment on the part of patients. The transducers are placed directly on the scalp, requiring patients to shave their head. Correct placement of the electrodes often necessitates the assistance of another person who also needs to undergo necessary training. Compliance issues can be partially alleviated by the fact that head coverings can be worn, disguising the transducers. Future advances in the device apparatus are expected to prolong battery life and decrease the size

TABLE 2. Post-hoc subgroup analyses on data derived from the study of Stupp et al., 2012*

\begin{tabular}{|c|c|c|c|c|c|c|c|}
\hline \multirow[b]{2}{*}{ Authors \& Year } & \multirow[b]{2}{*}{ Study Description } & \multicolumn{3}{|c|}{ Sample Size } & \multicolumn{3}{|c|}{ Median OS (mos) } \\
\hline & & TTF & Chemo Agent & Total & TTF & Chemo Agent & $\mathrm{p}$ Value \\
\hline \multirow[t]{2}{*}{ Ram et al., 2010} & $\begin{array}{l}\text { TTF monotherapy vs chemo in pts w/ favorable } \\
\text { prognostic characteristics }\end{array}$ & NR & NR & 110 & 8.8 & 6.6 & $<0.01$ \\
\hline & $\begin{array}{l}\text { TTF monotherapy vs chemo in pts who failed } \\
\text { bevacizumab }\end{array}$ & 23 & 21 & 44 & 4.4 & 3.1 & $<0.02$ \\
\hline Ram et al., 2011 & TTF monotherapy vs bevacizumab specifically & 120 & 36 & 156 & 6.6 & 5.0 & $0.048 \dagger$ \\
\hline Wong et al., 2011 & $\begin{array}{l}\text { Pts receiving }<1 \text { complete course of TTF } \\
\text { monotherapy vs chemo }\end{array}$ & 93 & 117 & 210 & 7.8 & 6.0 & 0.012 \\
\hline Kanner et al., 2013 & $\begin{array}{l}\text { TTF monotherapy vs chemo in pts }<60 \text { yrs of } \\
\text { age }\end{array}$ & NR & NR & 168 & 7.4 & 6.2 & 0.063 \\
\hline \multirow[t]{5}{*}{ Kanner et al., 2014} & $\begin{array}{l}\text { TTF monotherapy vs chemo for bevacizumab- } \\
\text { refractory glioblastoma }\end{array}$ & 23 & 21 & 44 & 6.0 & 3.3 & 0.016 \\
\hline & $\begin{array}{l}\text { TTF monotherapy vs chemo in pts w/ prior low- } \\
\text { grade glioma }\end{array}$ & 12 & 9 & 21 & 25.3 & 7.7 & 0.049 \\
\hline & $\begin{array}{l}\text { TTF monotherapy vs chemo in pts w/ tumor } \\
\text { size }>18 \mathrm{~cm}^{2}\end{array}$ & 39 & 41 & 80 & 5.6 & 3.3 & 0.009 \\
\hline & $\begin{array}{l}\text { TTF monotherapy vs chemo in pts w/ Karnof- } \\
\text { sky Performance Scale score }>80\end{array}$ & 83 & 77 & 160 & 7.9 & 6.1 & 0.045 \\
\hline & TTF monotherapy vs non-bevacizumab chemo & 120 & 81 & 201 & 6.6 & 6.6 & 0.586 \\
\hline
\end{tabular}

* Progression-free survival at 6 months and time to disease progression not included due to limited reporting by the reported investigations.

$\dagger$ Based on Cox proportional hazards model. 
and weight of the equipment, changes that will likely increase patients' compliance. Patients might also decide to discontinue therapy due to associated contact dermatitis commonly observed on the scalp. However, certain management techniques such as proper scalp washing and the use of topical antibiotics and steroids have already shown to effectively mitigate such adverse dermatological changes, ${ }^{35}$ promoting greater adherence. Nonetheless, physicians must consider these issues when deciding to incorporate AEF therapy to their patients' treatment plan.

Overall, it has been shown that compliance problems are common, as noted in the randomized control trial conducted by Stupp et al. ${ }^{65}$ in which more than $20 \%$ (27 of 120) of the patients originally assigned to the AEF cohort discontinued treatment earlier than expected. Thus, only 93 of the 120 patients completed a full cycle of AEF therapy. Importantly, median OS and PFS6 were significantly longer in these 93 patients who completed the full cycle compared with those patients receiving chemotherapy. ${ }^{27,80}$ Interestingly, in a post-hoc analysis conducted on this randomized clinical trial, compliance in patients below 60 years of age was statistically higher compared with those above 60 (80\% vs 74\%, respectively; $p=0.043) .{ }^{26}$ The importance of patient compliance cannot be overstated, as the results of the original randomized trial, ${ }^{65}$ as well as those of a previously mentioned pilot trial, ${ }^{30}$ demonstrated that the duration of AEF therapy was highly correlated with survival $\left(r^{2}=0.92\right) .{ }^{75}$ Further results also revealed that the patients in the trial who used the device for more than 18 hours daily also had improved survival compared with those who used the device less than 18 hours ( 7.7 vs 4.5 months, respectively; $\mathrm{p}=0.042) .{ }^{27}$

\section{Resistance to AEF Therapy}

Theoretically, long-term resistance to AEFs therapy is less likely to occur compared with standard chemotherapy, especially because no specific targeting of cell surface receptors occurs. Moreover, mitosis represents an inherent process within cancer cells necessary for proliferation. On the other hand, multiple-drug resistance in glioblastoma is a common phenomenon observed in patients treated with long-term chemotherapy. ${ }^{12,51}$ Additionally, the efficacy of chemotherapy agents is often limited due to the necessity that the therapeutic agent transverse the blood-brain barrier, a non-issue in AEF therapy. In accordance with such arguments, patients in the Phase III trial ${ }^{65}$ receiving AEF therapy experienced more favorable radiological responses, with only $14 \%$ of the patients demonstrating radiological changes suggesting emergence of resistance in the recurrent tumors.

One potential mechanism of resistance of glioblastomas to AEFs involves cell size. The initial studies ${ }^{28} \mathrm{dem}-$ onstrated that the optimal AEF frequency was inversely related to the cell size of the particular cancer line. Turner et al. ${ }^{70,71}$ further substantiate this theory, having reported a patient who developed a slow-growing lesion containing a giant cell glioblastoma with multiple syncytial-type cells following AEF and temozolomide treatment. This type of resistance can be potentially overcome by reducing the frequency of the emitted electric fields, as previously dem- onstrated in studies with ovarian cancer cells..$^{18}$ This finding suggests the importance of constant imaging monitoring (and sometimes even the necessity of new biopsies) to determine whether the parameters of the treatment with AEFs need to be adjusted. Moreover, as AEF therapy has been shown to induce genetic alterations of the tumor, ${ }^{37}$ it may be possible that resistance to AEFs may also occur by subsequent mutations in DNA repair mechanisms in the tumor cells.

Other research lines have attempted to increase the tumor response to AEFs by focusing on the current limitations of the applied electric fields rather than on the actual characteristics of the tumor. Specifically, Turner et al. ${ }^{71}$ suggest that a higher risk of treatment failure occurs near the margins of the tumor, where the strength of the electric field is reduced. Interestingly, recent studies have utilized MRI data to construct head models to more accurately assess the distribution of the oscillating electric fields in different areas of the brain. The obtained data suggest that the electric field strength is highest in the CSF, followed by white matter and then gray matter, ${ }^{42,78}$ a correlation that is directly related to relative impedance properties of such regions. ${ }^{86}$

\section{Contraindications to AEF Therapy}

Despite its limited systemic toxicity, AEF therapy is not recommended in certain patient populations. ${ }^{14}$ No studies to date have examined the safety profile of AEF therapy in patients with implanted electrical devices, including pacemakers, defibrillators, or deep brain stimulators due to the possibility of electrical interference. Interestingly, AEF therapy was well tolerated in a patient with a glioma who had a pre-existing ventriculoperitoneal shunt with a nonprogrammable valve. ${ }^{44}$ However, no studies up to now have evaluated the effects of AEFs in patients with programmable shunts. Additionally, any patients with a hypersensitivity to the hydrogel used for the placement of the scalp electrodes should not receive AEF therapy. Finally, as suggested above, patients unable to fully comply with the necessary continuity of AEF therapy or lacking the appropriate cognitive level or social support for successful compliance with the treatment recommendations will likely have a reduced benefit from this type of therapy.

\section{Future Perspectives}

It is expected that the use of AEFs as part of the multimodal treatment in patients with glioblastoma will continue to gain momentum following its FDA approval in 2011. Recent guidelines released by the National Comprehensive Cancer Network (2013) approve of the use of AEFs in patients with recurring or resistant tumors alongside chemotherapy options. ${ }^{45}$ Several clinical trials examining the use of AEFs in combination therapy with temozolomide and/or bevacizumab in the context of newly diagnosed ${ }^{13}$ or recurrent glioblastoma have been recently approved and are already recruiting participants. Additionally, a Phase II study has been initiated in order to evaluate the efficacy of AEFs in metastatic tumors from non-small cell lung cancer following stereotactic radiosurgery in comparison with radiosurgery alone. ${ }^{77}$ Future data as well as outcome analyses in 
patients treated with AEFs are expected to become more readily available through the continued development of the Patient Registry Dataset (PRiDe), a registry of all patients with recurrent glioblastoma who receive AEF therapy in the clinical setting since its FDA approval in $2011 .{ }^{43}$ Finally, future basic science investigations are warranted in order to determine the exact therapeutic mechanism of AEFs as well as to improve parameters for AEF delivery in order to achieve a more targeted application to the tumor zone.

In the future, clinical trials should also consider the therapeutic effects of AEF therapy in the context of the specific genetic composition (such as $\mathrm{O}^{6}$-methylguanine-DNA methyltransferase and isocitrate dehydrogenase mutations) and histological variations (such as the recently described proneural, neural, classical, and mesenchymal subtypes). This analysis may provide a better understanding of which particular subtypes of glioblastoma are more susceptible (or resistant) to AEF therapy. Eventually, these data could be used to "fine-tune" AEF frequencies to specific subtypes of tumor. Recurrent tumors will likely require a different applied frequency of AEFs, especially considering the molecular differences that have been noted between initial malignant tumors and their subsequent recurrences. ${ }^{59}$ Additionally, the efficacy of AEFs in neurooncology has currently only been established for glioblastoma; thus, further clinical studies investigating its potential application in other CNS tumors are necessary. Interestingly, one study did report a greater than $60 \%$ radiographic decrease in size of an incidental meningioma in a patient undergoing AEF therapy for a concurrent glioblastoma. ${ }^{60}$

Future studies must also continue to monitor the influence of AEFs on normal proliferating glial cells and permanent neuronal cell types. In previous studies AEFs have already been shown to affect neural stem cell viability and differentiation and proliferation, ${ }^{1,39}$ promote neuron cell body oscillation, ${ }^{50}$ and accelerate healing through neurite growth stimulation. ${ }^{22,46}$ Also, studies have shown that applied electric currents may modulate neuronal activity by altering firing rate, excitation properties, and spike timing. ${ }^{52,55,82}$ Therefore, the long-term cognitive effects of treatment with AEFs in terms of memory and learning should also be taken into account by future safety assessment studies. However, given the especially poor prognosis of glioblastoma as well as the promising results of therapy with AEFs, such issues should not prevent further exploration of this therapeutic modality in the clinical setting.

Finally, investigation regarding how AEFs could be combined with other standard treatment modalities (such as chemotherapy and radiation) is required in order to better delineate clinical protocols for newly diagnosed and recurrent glioblastoma designed to maximize a patient's prognosis and reduce overall side effects of multimodal treatment. For instance, enhanced effectiveness in AEF therapy is expected if it is incorporated with drugs that also alter the mitotic process, such as taxols and vinblastine. This synergism has already been noted in breast cancer cells when treated with paclitaxel and AEFs. ${ }^{29}$

\section{Conclusions}

Current cancer treatment protocols often incorporate multimodal therapeutic strategies including resection, radiation, and chemotherapy, as an attempt to increase treatment efficacy without increasing toxicity. Despite this approach, the prognosis of patients with glioblastoma remains relatively poor and adverse effects associated with treatment often compromise patients' quality of life. Preclinical data, pilot studies, case reports, and a recent Phase III, prospective randomized clinical trial support the use of AEFs in recurrent glioblastoma as having at least similar efficacy when compared with current chemotherapeutic regimens. Additionally AEF therapy has been shown to have a benign profile in terms of systemic adverse effects, allowing its use either as a monotherapy or in combination with chemotherapeutic agents, possibly in dose-reduced regimens, ultimately improving the overall toxicity profile of such therapies while maintaining efficacy rates.

Despite initial promising results (especially in pilot in vitro and in vivo studies), the clinical data examining the efficacy of AEFs remain limited. Comparatively, current radiation and chemotherapy regimens have been substantiated by several years of clinical investigations. Nonetheless, AEF therapy represents an entirely novel strategy in the treatment of brain tumors. In the future, fine-tuning of AEFs by adjusting field intensity, frequency, and direction (as well as novel forms of AEFs with more targeted delivery) may generate new possibilities for individualized treatment and improved clinical outcomes.

As the field of neurooncology continues to progress, new developments in currently available therapies as well as the introduction of novel strategies (such as AEFs) are expected to improve the outcomes of patients harboring tumors which, at the present stage, are still associated with dismal prognoses. Although AEFs are not expected to be a magic bullet for the cure of brain cancer, they do promise to become part of the multimodal therapies in neurooncology in the near future, which are expected to improve survival while minimizing side effects of current treatment strategies and increasing patients' quality of life.

\section{References}

1. Ariza CA, Fleury AT, Tormos CJ, Petruk V, Chawla S, Oh J, et al: The influence of electric fields on hippocampal neural progenitor cells. Stem Cell Rev 6:585-600, 2010

2. Bae SH, Park MJ, Lee MM, Kim TM, Lee SH, Cho SY, et al: Toxicity profile of temozolomide in the treatment of 300 malignant glioma patients in Korea. J Korean Med Sci 29:980-984, 2014

3. Bassett CA, Pilla AA, Pawluk RJ: A non-operative salvage of surgically-resistant pseudarthroses and non-unions by pulsing electromagnetic fields. A preliminary report. Clin Orthop Relat Res (124):128-143, 1977

4. Bermudez V, Capron N, Gase T, Gatti FG, Kajzar F, Leigh DA, et al: Influencing intramolecular motion with an alternating electric field. Nature 406:608-611, 2000

5. Bigner DD, Bigner SH, Pontén J, Westermark B, Mahaley MS, Ruoslahti E, et al: Heterogeneity of genotypic and phenotypic characteristics of fifteen permanent cell lines derived from human gliomas. J Neuropathol Exp Neurol 40:201229, 1981

6. Brem H, Piantadosi S, Burger PC, Walker M, Selker R, Vick NA, et al: Placebo-controlled trial of safety and efficacy of intraoperative controlled delivery by biodegradable polymers of chemotherapy for recurrent gliomas. Lancet 345:10081012,1995 
7. Burger PC, Green SB: Patient age, histologic features, and length of survival in patients with glioblastoma multiforme. Cancer 59:1617-1625, 1987

8. Chen H, Liu R, Liu J, Tang J: Growth inhibition of malignant melanoma by intermediate frequency alternating electric fields, and the underlying mechanisms. J Int Med Res 40:85-94, 2012

9. Darefsky AS, King JT Jr, Dubrow R: Adult glioblastoma multiforme survival in the temozolomide era: a populationbased analysis of Surveillance, Epidemiology, and End Results registries. Cancer 118:2163-2172, 2012

10. Dixit S, Hingorani M, Achawal S, Scott I: The sequential use of carmustine wafers (Gliadel $\left.{ }^{\circledR}\right)$ and post-operative radiotherapy with concomitant temozolomide followed by adjuvant temozolomide: a clinical review. Br J Neurosurg 25:459-469, 2011

11. Elzinga G, Wong ET: Resolution of cystic enhancement to add-on tumor treating electric fields for recurrent glioblastoma after incomplete response to bevacizumab. Case Rep Neurol 6:109-115, 2014

12. Eramo A, Ricci-Vitiani L, Zeuner A, Pallini R, Lotti F, Sette $\mathrm{G}$, et al: Chemotherapy resistance of glioblastoma stem cells Cell Death Differ 13:1238-1241, 2006

13. Farber O, Weinberg U, Palti Y: MR-008. Phase III trial of tumor treating fields (TTFields) together with temozolomide (TMZ) compared to temozolomide alone in patients with newly diagnosed glioblastoma (GBM). Neuro Oncol 15 (Suppl 3):iii76, 2013 (Abstract)

14. Fonkem E, Wong ET: NovoTTF-100A: a new treatment modality for recurrent glioblastoma. Expert Rev Neurother 12:895-899, 2012

15. Friedman HS, Prados MD, Wen PY, Mikkelsen T, Schiff D, Abrey LE, et al: Bevacizumab alone and in combination with irinotecan in recurrent glioblastoma. J Clin Oncol 27:47334740, 2009

16. Fritz J, Cooper EB, Gaudet S, Sorger PK, Manalis SR: Electronic detection of DNA by its intrinsic molecular charge. Proc Natl Acad Sci U S A 99:14142-14146, 2002

17. Gascoyne PR, Vykoukal J: Particle separation by dielectrophoresis. Electrophoresis 23:1973-1983, 2002

18. Giladi M, Porat Y, Schneiderman R, Munster M, Weinberg U, Kirson E, et al: ET-029. Overcoming cell size escape from tumor treating fields using a varying frequency treatment paradigm in-vitro. Neuro Oncol 15 (Suppl 3):iii43, 2013 (Abstract)

19. Giladi M, Tichon A, Schneiderman R, Porat Y, Munster M, Dishon M, et al: ET-028. Alternating electric fields (TTFields) inhibit DNA damage repair response in cancer cell lines. Neuro Oncol 15 (Suppl 3):iii43, 2013 (Abstract)

20. Giladi M, Wasserman Y, Urman N, Porat Y, Schneiderman R, Munster M, et al: MP-005. Meta-analysis of cancer cell line genomes based on their response to alternating electric fields (TTFields). Neuro Oncol 15 (Suppl 3):iii86, 2013 (Abstract)

21. Gitlin I, Carbeck JD, Whitesides GM: Why are proteins charged? Networks of charge-charge interactions in proteins measured by charge ladders and capillary electrophoresis. Angew Chem Int Ed Engl 45:3022-3060, 2006

22. Graves MS, Hassell T, Beier BL, Albors GO, Irazoqui PP: Electrically mediated neuronal guidance with applied alternating current electric fields. Ann Biomed Eng 39:17591767,2011

23. Hapani S, Sher A, Chu D, Wu S: Increased risk of serious hemorrhage with bevacizumab in cancer patients: a metaanalysis. Oncology 79:27-38, 2010

24. Hondroulis E, Melnick SJ, Zhang X, Wu ZZ, Li CZ: Electrical field manipulation of cancer cell behavior monitored by whole cell biosensing device. Biomed Microdevices 15:657663,2013
25. Johnson DR, O’Neill BP: Glioblastoma survival in the United States before and during the temozolomide era. J Neurooncol 107:359-364, 2012

26. Kanner AA, Wong ET, Villano JL, Ram Z, EF-11 Investigators: NO-065. Tumor treating fields (TTFields) in recurrent GBM. An updated subgroup analysis of the phase III data. Neuro Oncol 15 (Suppl 3):iii114, 2013 (Abstract)

27. Kanner AA, Wong ET, Villano JL, Ram Z, EF-11 Investigators: Post hoc analyses of intention-to-treat population in phase III comparison of NovoTTF-100A ${ }^{\mathrm{TM}}$ system versus best physician's choice chemotherapy. Semin Oncol 41 Suppl 6:S25-S34, 2014

28. Kirson ED, Dbalý V, Tovarys F, Vymazal J, Soustiel JF, Itzhaki A, et al: Alternating electric fields arrest cell proliferation in animal tumor models and human brain tumors. Proc Natl Acad Sci U S A 104:10152-10157, 2007

29. Kirson ED, Gurvich Z, Schneiderman R, Dekel E, Itzhaki A, Wasserman Y, et al: Disruption of cancer cell replication by alternating electric fields. Cancer Res 64:3288-3295, 2004

30. Kirson ED, Schneiderman RS, Dbalý V, Tovarys F, Vymazal J, Itzhaki A, et al: Chemotherapeutic treatment efficacy and sensitivity are increased by adjuvant alternating electric fields (TTFields). BMC Med Phys 9:1, 2009

31. Kirson ED, Wasserman Y, Izhaki A, Mordechovich D, Gurvich Z, Dbaly V, et al: NO-54. Modeling tumor growth kinetics and its implications for TTFields treatment planning. Neuro Oncol 12 (Suppl 4):iv48, 2010 (Abstract)

32. Kizilarslanoglu MC, Aksoy S, Yildirim NO, Ararat E, Sahin I, Altundag K: Temozolomide-related infections: review of the literature. J BUON 16:547-550, 2011

33. Kreisl TN, Kim L, Moore K, Duic P, Royce C, Stroud I, et al: Phase II trial of single-agent bevacizumab followed by bevacizumab plus irinotecan at tumor progression in recurrent glioblastoma. J Clin Oncol 27:740-745, 2009

34. Kristiansen K, Hagen S, Kollevold T, Torvik A, Holme I, Nesbakken R, et al: Combined modality therapy of operated astrocytomas grade III and IV. Confirmation of the value of postoperative irradiation and lack of potentiation of bleomycin on survival time: a prospective multicenter trial of the Scandinavian Glioblastoma Study Group. Cancer 47:649652,1981

35. Lacouture ME, Davis ME, Elzinga G, Butowski N, Tran D, Villano JL, et al: Characterization and management of dermatologic adverse events with the NovoTTF-100A System, a novel anti-mitotic electric field device for the treatment of recurrent glioblastoma. Semin Oncol 41 (Suppl 4):S1-S14, 2014

36. Laws ER, Parney IF, Huang W, Anderson F, Morris AM, Asher A, et al: Survival following surgery and prognostic factors for recently diagnosed malignant glioma: data from the Glioma Outcomes Project. J Neurosurg 99:467-473, 2003

37. Lee SX, Wong E, Swanson K: IR-022. Disruption of cell division within anaphase by tumor treating electric fields (TTFields) leads to immunogenic cell death. Neuro Oncol 15 (Suppl 3):iii66-iii67, 2013 (Abstract)

38. Louis DN, Ohgaki H, Wiestler OD, Cavenee WK, Burger PC, Jouvet A, et al: The 2007 WHO classification of tumours of the central nervous system. Acta Neuropathol 114:97-109, 2007

39. Matos MA, Cicerone MT: Alternating current electric field effects on neural stem cell viability and differentiation. Biotechnol Prog 26:664-670, 2010

40. McGirt MJ, Than KD, Weingart JD, Chaichana KL, Attenello FJ, Olivi A, et al: Gliadel (BCNU) wafer plus concomitant temozolomide therapy after primary resection of glioblastoma multiforme. J Neurosurg 110:583-588, 2009

41. McIntosh JR, Grishchuk EL, West RR: Chromosome-microtubule interactions during mitosis. Annu Rev Cell Dev Biol 18:193-219, 2002 
42. Miranda PC, Mekonnen A, Salvador R, Basser PJ: Predicting the electric field distribution in the brain for the treatment of glioblastoma. Phys Med Biol 59:4137-4147, 2014

43. Mrugala MM, Engelhard HH, Dinh Tran D, Kew Y, Cavaliere R, Villano JL, et al: Clinical practice experience with NovoTTF-100A ${ }^{\mathrm{TM}}$ system for glioblastoma: the Patient Registry Dataset (PRiDe). Semin Oncol 41 Suppl 6:S4-S13, 2014

44. Mrugala MM, Graham CA, Rockhill JK, Silbergeld DL: P17.60. Novo-TTF 100A system used successfully in a patient with a ventriculo-peritoneal shunt. Neuro Oncol 16 (Suppl 2):ii101, 2014 (Abstract)

45. National Comprehensive Cancer Network: NCCN Clinical Practice Guidelines in Oncology (NCCN Guidelines ${ }^{\circledR}$ ). Central nervous system cancers. Version 2.2014. Fort Washington, PA: National Comprehensive Cancer Network, 2014

46. Nguyen HT, Sapp S, Wei C, Chow JK, Nguyen A, Coursen $\mathrm{J}$, et al: Electric field stimulation through a biodegradable polypyrrole-co-polycaprolactone substrate enhances neural cell growth. J Biomed Mater Res A 102:2554-2564, 2014

47. Norden AD, Young GS, Setayesh K, Muzikansky A, Klufas $\mathrm{R}$, Ross GL, et al: Bevacizumab for recurrent malignant gliomas: efficacy, toxicity, and patterns of recurrence. Neurology 70:779-787, 2008

48. Nuccitelli R, Pliquett U, Chen X, Ford W, Swanson RJ, Beebe SJ, et al: Nanosecond pulsed electric fields cause melanomas to self-destruct. Biochem Biophys Res Commun 343:351360, 2006

49. Ostrom QT, Gittleman H, Liao P, Rouse C, Chen Y, Dowling J, et al: CBTRUS statistical report: primary brain and central nervous system tumors diagnosed in the United States in 2007-2011. Neuro Oncol 16 Suppl 4:iv1-iv63, 2014

50. Pan L, Cirillo J, Borgens RB: Neuronal responses to an asymmetrical alternating current field can mimic those produced by an imposed direct current field in vitro. $\mathbf{J}$ Neurosci Res 90:1522-1532, 2012

51. Quezada C, Garrido W, Oyarzún C, Fernández K, Segura $\mathrm{R}$, Melo R, et al: 5'-ectonucleotidase mediates multiple-drug resistance in glioblastoma multiforme cells. J Cell Physiol 228:602-608, 2013

52. Radman T, Su Y, An JH, Parra LC, Bikson M: Spike timing amplifies the effect of electric fields on neurons: implications for endogenous field effects. J Neurosci 27:3030-3036, 2007

53. Ram Z, Gutin PH, Stupp R: NO-55. Subgroup and quality of life analyses of the phase III clinical trial of NovoTTF-100A versus best standard chemotherapy for recurrent glioblastoma. Neuro Oncol 12 (Suppl 4):iv48-iv49, 2010 (Abstract)

54. Ram Z, Wong ET, Gutin PH: NO-50. Comparing the effect of NovoTTF to bevacizumab in recurrent GBM: a post-hoc subanalysis of the phase III trial data. Neuro Oncol 13 (Suppl 3):iii52, 2011 (Abstract)

55. Reato D, Rahman A, Bikson M, Parra LC: Effects of weak transcranial alternating current stimulation on brain activity-a review of known mechanisms from animal studies. Front Hum Neurosci 7:687, 2013

56. Rulseh AM, Keller J, Klener J, Sroubek J, Dbalý V, Syrůček $\mathrm{M}$, et al: Long-term survival of patients suffering from glioblastoma multiforme treated with tumor-treating fields. World J Surg Oncol 10:220, 2012

57. Salvati M, D'elia A, Frati A, Brogna C, Santoro A, Delfini R: Safety and feasibility of the adjunct of local chemotherapy with biodegradable carmustine (BCNU) wafers to the standard multimodal approach to high grade gliomas at first diagnosis. J Neurosurg Sci 55:1-6, 2011

58. Sauer H, Bekhite MM, Hescheler J, Wartenberg M: Redox control of angiogenic factors and CD31-positive vessel-like structures in mouse embryonic stem cells after direct current electrical field stimulation. Exp Cell Res 304:380-390, 2005

59. Saxena A, Shriml LM, Dean M, Ali IU: Comparative mo- lecular genetic profiles of anaplastic astrocytomas/glioblastomas multiforme and their subsequent recurrences. Oncogene 18:1385-1390, 1999

60. Schaff L, Armentano F, Harrison C, Lassman A, McKhann G, Iwamoto F: NO-006. Radiographic response of an incidental meningioma in a patient with glioblastoma on NovoTTF therapy. Neuro Oncol 15 (Suppl 3):iii99, 2013 (Abstract)

61. Siegel R, Ma J, Zou Z, Jemal A: Cancer statistics, 2014. CA Cancer J Clin 64:9-29, 2014

62. Sisken BF, Walker J, Orgel M: Prospects on clinical applications of electrical stimulation for nerve regeneration. J Cell Biochem 51:404-409, 1993

63. Stupp R, Hegi ME, Mason WP, van den Bent MJ, Taphoorn MJ, Janzer RC, et al: Effects of radiotherapy with concomitant and adjuvant temozolomide versus radiotherapy alone on survival in glioblastoma in a randomised phase III study: 5-year analysis of the EORTC-NCIC trial. Lancet Oncol 10:459-466, 2009

64. Stupp R, Mason WP, van den Bent MJ, Weller M, Fisher $\mathrm{B}$, Taphoorn MJ, et al: Radiotherapy plus concomitant and adjuvant temozolomide for glioblastoma. N Engl J Med 352:987-996, 2005

65. Stupp R, Wong ET, Kanner AA, Steinberg D, Engelhard H, Heidecke V, et al: NovoTTF-100A versus physician's choice chemotherapy in recurrent glioblastoma: a randomised phase III trial of a novel treatment modality. Eur J Cancer 48:2192-2202, 2012

66. Syrigos KN, Karapanagiotou E, Boura P, Manegold C, Harrington K: Bevacizumab-induced hypertension: pathogenesis and management. BioDrugs 25:159-169, 2011

67. Traitcheva N, Berg H: Electroporation and alternating current cause membrane permeation of photodynamic cytotoxins yielding necrosis and apoptosis of cancer cells. Bioelectrochemistry 79:257-260, 2010

68. Trakic A, Akhand M, Wang H, Mason D, Liu F, Wilson S, et al: Computational modelling of blood-flow-induced changes in blood electrical conductivity and its contribution to the impedance cardiogram. Physiol Meas 31:13-33, 2010

69. Trock DH, Bollet AJ, Markoll R: The effect of pulsed electromagnetic fields in the treatment of osteoarthritis of the knee and cervical spine. Report of randomized, double blind, placebo controlled trials. J Rheumatol 21:1903-1911, 1994

70. Turner S, Gergel T, Lacroix M, Toms S: NO-134. The effect of field strength on GBM response in patients treated with Novocure-TTF. Neuro Oncol 15 (Suppl 3):iii131, 2013 (Abstract)

71. Turner SG, Gergel T, Wu H, Lacroix M, Toms SA: The effect of field strength on glioblastoma multiforme response in patients treated with the NovoTTF ${ }^{\mathrm{TM}}-100$ A system. World J Surg Oncol 12:162, 2014

72. US Food and Drug Administration: NovoTTF-100A System - P100034. (http://www.fda.gov/MedicalDevices/ProductsandMedicalProcedures/DeviceApprovalsandClearances/ Recently-ApprovedDevices/ucm254480.htm) [Accessed January 18, 2015]

73. Villano JL, Williams LE, Watson KS, Ignatius N, Wilson MT, Valyi-Nagy T, et al: Delayed response and survival from NovoTTF-100A in recurrent GBM. Med Oncol 30:338, 2013

74. Vredenburgh JJ, Desjardins A, Herndon JE II, Marcello J, Reardon DA, Quinn JA, et al: Bevacizumab plus irinotecan in recurrent glioblastoma multiforme. J Clin Oncol 25:4722-4729, 2007

75. Vymazal J, Wong ET: Response patterns of recurrent glioblastomas treated with tumor treating fields (TTFields). Semin Oncol 41 Suppl 6:S14-S24, 2014

76. Walker MD, Alexander E Jr, Hunt WE, MacCarty CS, Mahaley MS Jr, Mealey J Jr, et al: Evaluation of BCNU and/or radiotherapy in the treatment of anaplastic gliomas. A cooperative clinical trial. J Neurosurg 49:333-343, 1978 
77. Weinberg U, Farber O, Palti Y: MR-036. A phase II randomized study of TTFields therapy versus supportive care in non-small cell lung cancer (NSCLC) patients with 1-3 brain metastases (BM) following stereotactic radio-surgery (SRS). Neuro Oncol 15 (Suppl 3):iii84, 2013 (Abstract)

78. Wenger C, Miranda PC, Mekonnen A, Salvador R, Basser P: TM-028. Electric fields for the treatment of glioblastomas: a modeling study. Neuro Oncol 15 (Suppl 3):iii241, 2013 (Abstract)

79. Wong ET, Elzinga G, Chung A, Barron L, Bloom J, Swanson $\mathrm{KD}$ : NO-146. Objective response in recurrent glioblastoma from adjuvant NovoTTF-100A and TCCC after temozolomide and bevacizumab failure. Neuro Oncol 15 (Suppl 3):iii134, 2013 (Abstract)

80. Wong ET, Ram Z, Gutin PH, Stupp R: OT-09. Updated survival data of the phase III clinical trial of NovoTTF-100A versus best standard chemotherapy for recurrent glioblastoma. Neuro Oncol 13 (Suppl 3):iii87, 2011 (Abstract)

81. Wu S, Kim C, Baer L, Zhu X: Bevacizumab increases risk for severe proteinuria in cancer patients. J Am Soc Nephrol 21:1381-1389, 2010

82. Xu W, Wolff BS, Wu JY: Low-intensity electric fields induce two distinct response components in neocortical neuronal populations. J Neurophysiol 112:2446-2456, 2014

83. Yung WK, Prados MD, Yaya-Tur R, Rosenfeld SS, Brada M, Friedman HS, et al: Multicenter phase II trial of temozolomide in patients with anaplastic astrocytoma or anaplastic oligoastrocytoma at first relapse. J Clin Oncol 17:2762-2771, 1999

84. Zhao M: Electrical fields in wound healing-an overriding signal that directs cell migration. Semin Cell Dev Biol 20:674-682, 2009

85. Zhao M, Forrester JV, McCaig CD: A small, physiological electric field orients cell division. Proc Natl Acad Sci U S A 96:4942-4946, 1999

86. Zrinzo L, Hariz MI: Impedance recording in functional neurosurgery, in Lozano AM, Gildenberg PL, Tasker RR (eds): Textbook of Stereotactic and Functional Neurosurgery, ed 2. Berlin: Springer, 2009, Vol 1, pp 1325-1330

\section{Author Contributions}

Conception and design: Mattei, Rehman. Acquisition of data: all authors. Analysis and interpretation of data: Rehman, Elmore. Drafting the article: Rehman, Elmore. Critically revising the article: Mattei. Reviewed submitted version of manuscript: all authors. Approved the final version of the manuscript on behalf of all authors: Mattei. Study supervision: Mattei.

\section{Correspondence}

Tobias A. Mattei, Department of Neurosurgery, Invision Health Brain \& Spine Center, 400 International Dr., Williamsville, NY 14421.email: tobiasmattei@yahoo.com. 